\title{
Changing Pattern of Esophageal Cancer Incidence in New Mexico: A 30-Year Evaluation
}

\author{
Kenneth J. Vega · M. Mazen Jamal • \\ Charles L. Wiggins
}

Received: 2 April 2009/ Accepted: 9 July 2009/Published online: 18 August 2009

(c) The Author(s) 2009. This article is published with open access at Springerlink.com

\begin{abstract}
Background and Aim The incidence of esophageal adenocarcinoma has increased over the last 30 years, especially in non-Hispanic whites (nHw). Recent work indicates an increase in Hispanic Americans (HA). It is important to understand the effect of ethnicity on cancer occurrence over a prolonged interval.

Methods We searched the New Mexico Tumor Registry for all cases of esophageal cancer from 1 January 1973 to 31 December 2002. Inclusion criteria were histologic diagnosis of adenocarcinoma or squamous cell carcinoma, ethnicity and gender. Incidence rates for both were compared among ethnic groups in 5-year intervals.

Results Nine hundred eighty-eight patients met the criteria. Esophageal adenocarcinoma incidence rates $/ 100,000$ population increased significantly over 30 years; 1973 1977, 0.4 cases; 1978-1982, 0.4 cases; 1983-1987, 0.6 cases; 1988-1992, 1.2 cases, 1993-1997, 1.6 cases and 1998-2002, 2.2 cases; $P<0.001$. Squamous cell carcinoma incidence rates remained unchanged during the
\end{abstract}

K. J. Vega $(\bowtie)$

Division of Gastroenterology, University of Florida Health

Science Center/Jacksonville, 4555 Emerson Expressway,

Suite 300, Jacksonville, FL 32207, USA

e-mail: kenneth.vega@jax.ufl.edu

M. M. Jamal

Section of Gastroenterology, VAMC Long Beach, Long Beach, CA, USA

M. M. Jamal

University of California, Irvine, Irvine, CA, USA

C. L. Wiggins

New Mexico Tumor Registry, University of New Mexico, Albuquerque, NM, USA interval. In $\mathrm{nHw}$ and $\mathrm{HA}$, adenocarcinoma incidence rates increased significantly during the study period. In all minority groups, squamous cell carcinoma remained the major type.

Conclusions Esophageal adenocarcinoma incidence among nHw and HA increased from 1973 to 2002 in New Mexico. Squamous cell carcinoma remains predominant in minorities. Ethnicity may influence the histology or indicate an increased risk for certain types of esophageal cancer.

Keywords Esophageal cancer .

Adenocarcinoma of the esophagus .

Squamous cell carcinoma of the esophagus - Ethnicity ·

Race

\section{Introduction}

Esophageal cancer is the ninth leading cause of cancerrelated death in the US and is expected to result in 14,000 fatalities in 2008 [1]. Approximately, $75 \%$ of patients with esophageal cancer expire in the 1 st year post-diagnosis, and the 5-year survival rate is only $8 \%$ despite treatment. During the past 30 years, multiple investigators have demonstrated that the incidence of adenocarcinoma of the esophagus (ADENO) in the US and Europe has significantly increased compared with squamous cell carcinoma of the esophagus (SCCA) [1-11]. This increase has been observed primarily in non-Hispanic white males and in those of higher socioeconomic status $[1-3,8,9]$. It is well accepted that chronic gastroesophageal reflux disease and Barrett's esophagus are conditions associated with the increasing incidence of ADENO [10-15]. Other data indicate that Arizona nonHispanic whites and Hispanic Americans have the same prevalence of Barrett's esophagus [16]. 
Since esophageal cancer incidence varies within geographical regions of the US and the world, specific data on minority groups are indispensable in documenting whether epidemiological changes in esophageal cancer can be generalized. Multiple studies have confirmed that adenocarcinoma and Barrett's esophagus are uncommon among African Americans [3, 17-22]. Recently, Hispanic Americans have been similarly evaluated for esophageal cancer [18-21]. However, only one study has assessed esophageal cancer in Hispanic Americans for a period longer than 7 years [18].

The population of New Mexico is a diverse multiethnic group with four distinct groups: $45 \%$ non-Hispanic white, 42\% Hispanic, 10\% Native American and 3\% African American or other per the 2000 US census [23]. Data on esophageal cancer statewide are maintained in a single database. We retrospectively evaluated the incidence of esophageal cancer in New Mexico over a 30-year period in order to assess any continued change in incidence and to further evaluate the effect of ethnicity on esophageal cancer histology.

\section{Methods}

We retrospectively reviewed the New Mexico Tumor Registry to obtain all cases of esophageal carcinoma reported between 1973 and 2002. The New Mexico Tumor Registry identifies cancer cases through active surveillance of different medical information sources, including hospital records, outpatient clinic records, pathology and autopsy reports, death records and radiation therapy records. Once identified, information is collected on patient demographics and cancer characteristics and entered into a centralized database, which is part of the SEER (Surveillance, Epidemiology and End Results) cancer incidence database. The tumor registry database was reviewed for individual patient inclusion into the study. Inclusion criteria were selfreported ethnicity as non-Hispanic white, Hispanic American, Native American or African American and histologic diagnosis of ADENO or SCCA of the esophagus. Individual case information was gathered from the tumor registry abstract using a structured data abstraction protocol created prior to data collection. Clinical information included age at diagnosis, year of diagnosis and gender. Cases were placed into 65 -year time intervals for analysis: 1973-1977, 1978-1982, 1983-1987, 1988-1992, 1993-1997 and 1998-2002.

The overall and race-specific incidence rates were adjusted using the direct standardization method to reflect the age distribution of the 2000 US population. Incidence rates for the 5-year periods were expressed as proportional rates per 100,000 individuals. Incidence rates of squamous cell carcinoma and adenocarcinoma of the esophagus within different ethnic groups for 5-year periods were compared using Fisher's exact test. A result was considered statistically significant if $P<0.05$.

\section{Results}

A total of 1,168 patients with esophageal cancer were reported in New Mexico during the 30-year study period according to the tumor registry. One hundred eighty patients were excluded for the following reasons: histology not specified or histology other then ADENO or SCCA, and ethnicity other than non-Hispanic white, African, Hispanic or Native American. The remaining 988 patients met inclusion criteria and comprised the study group.

Demographic data are shown in Tables 1 and 2. Patient age ranged from 32 to 95 years. Of 988 cases of esophageal cancer, $527(53 \%)$ were SCCA, and 461 (47\%) were ADENO. Males represented $74 \%$ of the overall study group, $68 \%$ of the SCCA cases and $85 \%$ of the ADENO cases. Ethnic breakdown was as follows: non-Hispanic white 70\%, Hispanic Americans 23\%, African Americans $4 \%$ and Native Americans 3\%. There was no statistically significant difference in ethnic breakdown between those included and excluded from the investigation.

The incidence rates of esophageal cancer per 100,000 are seen in Figs. 1 and 2. ADENO of the esophagus

Table 1 Esophageal cancer characteristics in New Mexico, 19732002

\begin{tabular}{lccc}
\hline & \multicolumn{3}{l}{ Esophageal cancer cases } \\
\cline { 2 - 4 } & Total & SCCA & ADENO \\
\hline All ethnic groups $(N)$ & 988 & 527 & 461 \\
Non-Hispanic white $N(\%)$ & $707(71.6)$ & $329(62.4)$ & $378(82)$ \\
Hispanic American $N(\%)$ & $223(22.6)$ & $147(27.9)$ & $76(16.5)$ \\
African-American $N(\%)$ & $32(3.2)$ & $29(5.5)$ & $3(0.7)$ \\
Native-American $N(\%)$ & $26(2.6)$ & $22(4.2)$ & $4(0.8)$ \\
\hline
\end{tabular}

Table 2 Gender and histology distribution of esophageal cancer in New Mexico from 1973-2002

\begin{tabular}{lcccccc}
\hline & \multicolumn{2}{c}{ Squamous cell carcinoma } & & \multicolumn{2}{c}{ Adenocarcinoma } \\
\cline { 2 - 3 } \cline { 5 - 6 } \cline { 5 - 6 } & Male & Female & & Male & Female \\
\hline All ethnic groups & 355 & 172 & & 402 & 59 \\
Non-Hispanic white & 201 & 128 & & 328 & 50 \\
Hispanic-American & 114 & 33 & & 69 & 7 \\
African-American & 21 & 8 & & 2 & 1 \\
Native-American & 19 & 3 & & 3 & 1 \\
\hline
\end{tabular}




\section{Adenocarcinoma}

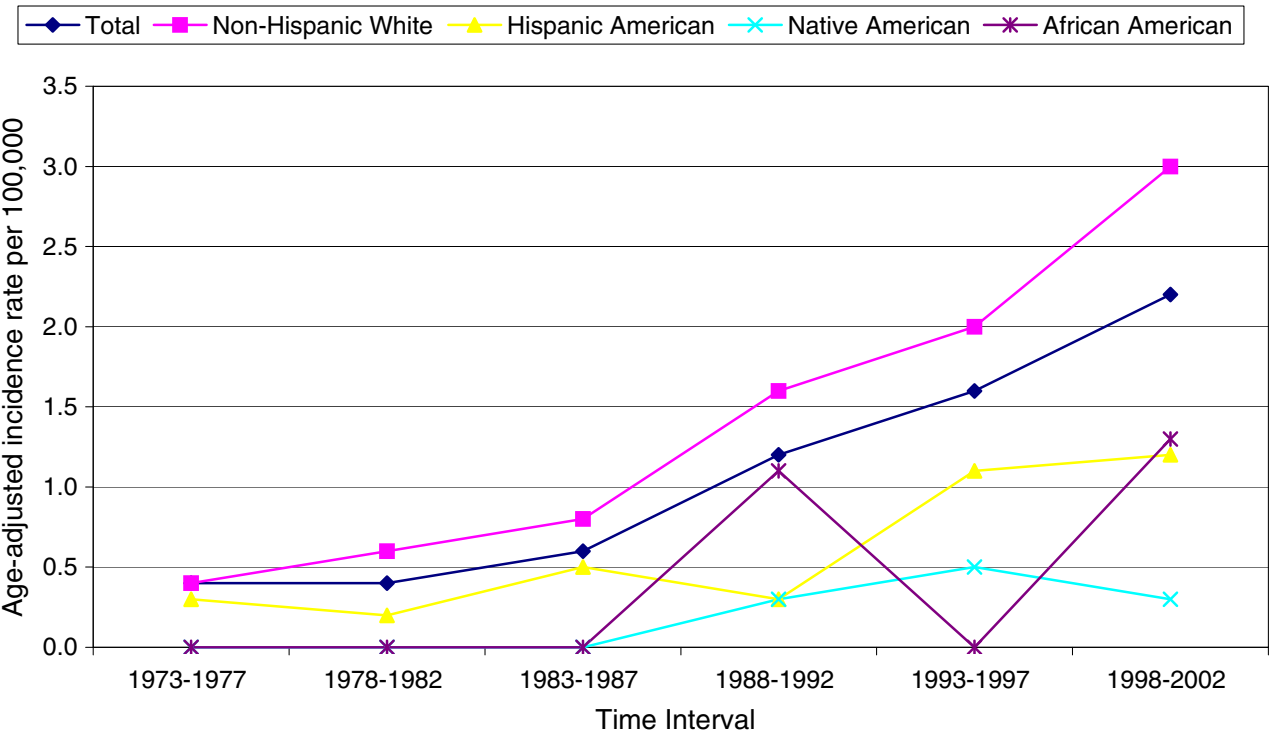

Fig. 1 Total population and ethnic incidence rates of esophageal adenocarcinoma in New Mexico. Total cases of the esophageal adenocarcinoma increased significantly over the 30-year period: 1973-1977, 0.4 cases per 100,000; 1978-1982, 0.4 cases per 100,000; $1983-1987,0.6$ cases per 100,$000 ; 1988-1992,1.2$ cases per 100,000 ;
1992-1997, 1.6 cases per 100,$000 ; 1998-2002,2.2$ cases per 100,000 $(P<0.001)$. Incidence rates increased over time in the non-Hispanic white, Hispanic and Native American populations. The increase seen in non-Hispanic whites and Hispanic Americans achieved statistical significance $(P<0.001$ for both groups)

\section{Squamous Cell Carcinoma}

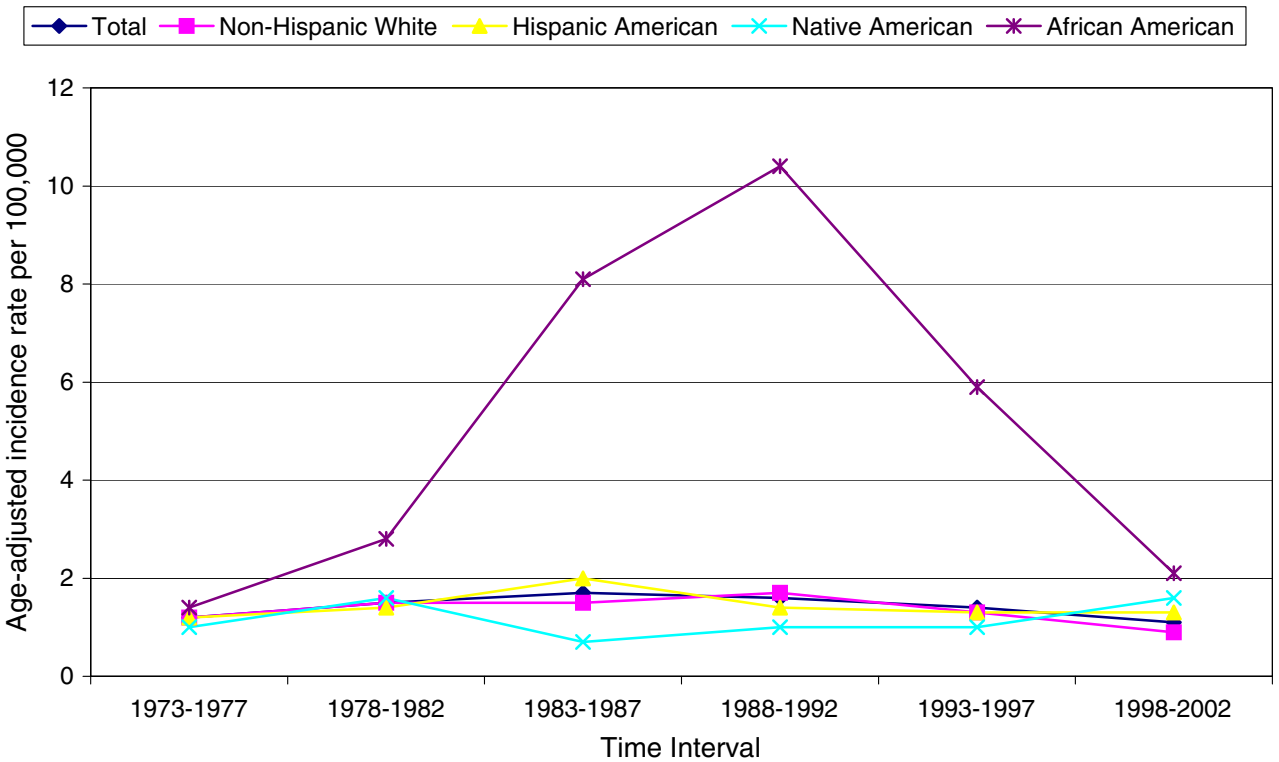

Fig. 2 Total and ethnic incidence rates of esophageal squamous cell carcinoma in New Mexico. Incidence rates did not vary significantly among the total population or between ethnic groups over time

increased significantly over the 30-year period: 1973-1977, 0.4 cases per 100,000 population; $1978-1982,0.4$ cases per 100,$000 ; 1983-1987,0.6$ cases per 100,$000 ; 1988-1992$, 1.2 cases per 100,000, 1992-1997, 1.6 cases per 100,000; 1998-2002, 2.2 cases per $100,000 \quad(P<0.001)$. In comparison, the incidence of SCCA per 100,000 population did not increase or decrease significantly over the study period: 1973-1977, 1.2 cases; 1978-1982, 1.5 cases; 1983-1987, 1.7 cases; 1988-1992, 1.6 cases; 1993-1997, 1.4 cases; 1998-2002, 1.1 cases. 
Time trends in the incidence of esophageal cancer by histology and ethnicity are also illustrated in Figs. 1 and 2. In the non-Hispanic white population, the incidence of ADENO increased significantly over the investigation: 1973-1977, 0.4 cases per 100,000; 1978-1982, 0.6 cases per 100,000 ; 1983-1987, 0.8 cases per 100,000 ; 19881992, 1.6 cases per 100,000; 1993-1997, 2 cases per 100,$000 ; 1998-2002$, 3 cases per $100,000(P<0.001$, Fig. 1). Also, the predominant histologic type seen in nonHispanic whites changed over the study period. During the four periods between 1973 and 1992, SCCA was the prevailing tumor histology. However, during the 1993-1997 and 1998-2002 periods, ADENO became the prevailing histology seen, and this change was statistically significant $(P<0.001$, Fig. 1 and 2). In Hispanics, a significant increase in incidence rate for ADENO was seen during the 1993-1997 and 1998-2002 intervals in comparison to all others: 1973-1977, 0.3 cases per 100,000; 1978-1982, 0.2 cases per 100,000; 1983-1987, 0.5 cases per 100,000; 1988-1992, 0.3 cases per 100,000; 1993-1997, 1.1 cases per 100,$000 ; 1998-2002,1.2$ cases per 100,000 $(P<0.001$, Fig. 1). Among all minorities, SCCA remained the predominant histologic type during all study periods (Fig. 2). Therefore, in New Mexico, the incidence of ADENO increased during the 30-year period, and this increase occurred primarily in non-Hispanic whites and to a lesser extent in Hispanics.

\section{Discussion}

Esophageal cancer continues to be one of the most lethal diseases in the US with limited survival and high mortality [1]. During the past 30 years, the incidence of esophageal ADENO has increased in comparison to SCCA, and this has occurred predominantly among non-Hispanic white males [1-9, 17-21]. Our study supports the present literature indicating that ADENO is rapidly increasing in nonHispanic whites, that it is increasing to a lesser degree in Hispanic Americans and that SCCA continues to be the primary type seen among African Americans, Hispanic Americans and Native Americans.

The present investigation is important since it continues to confirm a role for ethnicity as a determining factor in esophageal cancer within specific populations. In multiple investigations to date, esophageal adenocarcinoma is predominantly seen in non-Hispanic whites, SCCA remains predominant in African Americans, and a significant increase of esophageal adenocarcinoma in Hispanic Americans has been noted [3, 9, 17-21]. The present study indicates a protracted increase in esophageal adenocarcinoma incidence rate among both non-Hispanic whites and Hispanic Americans.
The demographic results of our study have both similarities and differences in comparison to the previous investigations due to the nature of the New Mexico population. The total number of cases observed is similar to previous work [3]. However, the ethnic breakdown of the minority population evaluated here, especially the Hispanic and Native American groups, has only been reported in one previous study [18]. The histologic variations noted are consistent with prior studies indicating an increased proportion of non-Hispanic whites with adenocarcinoma and other ethnic groups with squamous cell carcinoma predominating. This investigation has also confirmed a recently recognized occurrence: the increase of esophageal adenocarcinoma in the Hispanic American population [18, $19,21,22]$. However, both the prolonged rise of adenocarcinoma incidence within Hispanic Americans and the decreased incidence of SCCA among African Americans has not been reported to date.

Barrett's esophagus is an accepted risk factor for esophageal adenocarcinoma, but has been documented infrequently in minority groups [3, 16, 17, 22]. Among Hispanics, such information is only available in two investigations [16, 22]. In one study, Bersentes et al. [16] reported that there was no difference in the prevalence of Barrett's esophagus between non-Hispanic whites and Hispanics with or without gastroesophageal reflux disease seen at the Veterans Affairs Medical Center in Tucson, AZ. Recently, Abrams and associates noted Hispanics from the New York City area had a decreased prevalence of Barrett's esophagus at endoscopy [22]. Unfortunately, the New Mexico tumor registry database does not provide information regarding the presence or absence of Barrett's esophagus in the cases evaluated by the present investigation.

There are potential limitations to using the New Mexico tumor registry as a data source. First, the presence or absence of Barrett's esophagus was not reported. Second, no specific information pertaining to risk factors such as smoking, alcohol use, obesity or frequency of gastroesophageal reflux disease was available. In addition, the database may be subject to errors in reporting and entering patient information.

In contrast, there are strengths to the present investigation. The New Mexico tumor registry is part of the SEER cancer incidence database used for reporting cancer trends within the US. The catchment area for the New Mexico registry represents all areas of the state with a bias toward urban regions. Demonstration of opposing ethnic trends in different histologic types of esophageal cancer in the same population confers internal validity to the study results.

In summary, the recently reported changes in the incidence of esophageal adenocarcinoma and squamous cell carcinoma have been confirmed in the New Mexico 
population. Also, the previously noted significant increase in adenocarcinoma cases among Hispanic Americans of New Mexico from 1992-1997 has continued during the 1998-2002 period. As well, a decreased rate of SCCA among African Americans has been observed. The reasons for the predisposition of African, Hispanic and Native Americans developing squamous cell cancer of the esophagus, decreasing incidence of SCCA among African Americans along with the continued increase of adenocarcinoma incidence among New Mexico Hispanic Americans is not presently known. This suggests that ethnicity may influence esophageal cancer histology or that ethnic background can place an individual at increased risk for certain types of esophageal cancer. Notwithstanding, ethnicity either directly influences the type of esophageal cancer in Americans or may represent a surrogate marker for an as yet undefined risk factor in esophageal cancer. Further investigation and ethnic-specific research and education are needed to completely understand esophageal cancer in New Mexico and the US.

Open Access This article is distributed under the terms of the Creative Commons Attribution Noncommercial License which permits any noncommercial use, distribution, and reproduction in any medium, provided the original author(s) and source are credited.

\section{References}

1. Jemal A, Siegel R, Ward E, et al. Cancer Statistics, 2008. CA. Cancer J Clin. 2008;52:71-96. doi:10.3322/CA.2007.0010.

2. Blot WJ, Devesa SS, Kneller RW, et al. Rising incidence of adenocarcinoma of the esophagus and gastric cardia. JAMA. 1991;265:1287-1289. doi:10.1001/jama.265.10.1287.

3. Chen MYM, Ott DJ, Gelfand DW. More evidence for the increasing prevalence of adenocarcinoma of the esophagus over an 18-year period. J Clin Gastroenterol. 1995;21:254-262. doi: 10.1097/00004836-199510000-00019.

4. McKinney PA, Sharp L, MacFarlane GJ, et al. Oesophageal and gastric cancer in Scotland 1960-1990. Br J Cancer. 1994;63: 412-415.

5. Moyana TN, Janoski M. Recent trends in the epidemiology of esophageal cancer. Ann Clin Lab Sci. 1996;26:480-486.

6. Pera M, Cameron AJ, Trastek VF, et al. Increasing incidence of adenocarcinoma of the esophagus and esophagogastric junction. Gastroenterology. 1993;104:510-513.

7. Powell J, McConkey CC. Increasing incidence of adenocarcinoma of the gastric cardia and adjacent sites. $\mathrm{Br} J$ Cancer. 1990;59:440-443.
8. Yang PC, Davis S. Incidence of cancer of the esophagus in the US by histologic type. Cancer. 1988;92:612-617. doi:10.1002/ 1097-0142(19880201)61:3<612::AID-CNCR2820610332>3.0.CO; 2-Q.

9. Firoozi B, Vega KJ, Holland BK, et al. Epidemiologic pattern of esophageal cancer at an inner-city university hospital. J Assoc Acad Min Phys. 1999;10:44-48.

10. Winters C Jr, Spurling TJ, Chobanian SJ, et al. Barrett's esophagus: a prevalent, occult complication of gastroesophageal reflux disease. Gastroenterology. 1987;92:118-124.

11. Mann NS, Tsai MF, Nair PK. Barrett's esophagus in patients with symptomatic reflux esophagitis. Am J Gastroenterol. 1989;84: 1494-1496.

12. Cameron AJ, Kamath PS, Carpenter HC. Barrett's esophagus, the prevalence of short and long segments in reflux patients. Gastroenterology. 1995;108:A65. doi:10.1016/0016-5085(95)2292 2-1. Abstract.

13. Williamson WA, Ellis FH Jr, Gibb SP, et al. Barrett's esophagus. Prevalence and incidence of adenocarcinoma. Arch Intern Med. 1991;151:2212-2216. doi:10.1001/archinte.151.11.2212.

14. Tytgat GNJ. Does endoscopic surveillance in esophageal columnar metaplasia (Barrett's esophagus) have any real value? Endoscopy. 1995;27:19-26. doi:10.1055/s-2007-1005627.

15. Cameron AJ, Ott BJ, Payne WS. The incidence of adenocarcinoma in columnar lined (Barrett's) esophagus. $N$ Engl $J$ Med. 1985;313:857-859.

16. Bersentes K, Fass R, Padda S, et al. Prevalence of Barrett's esophagus in Hispanics is similar to Caucasians. Dig Dis Sci. 1998;43:1038-1041. doi:10.1023/A:1018834902694.

17. Chalasani N, Wo JM, Waring JP. Racial differences in the histology, location and risk factors of esophageal cancer. J Clin Gastroenterol. 1998;26:11-13. doi:10.1097/00004836-1998010 00-00004.

18. Vega KJ, Jamal MM. Changing pattern of esophageal cancer incidence in New Mexico. Am J Gastroenterol. 2000;95:23522356. doi:10.1111/j.1572-0241.2000.02329.x.

19. Kubo A, Corley DA. Marked multi-ethnic variation of esophageal and gastric cardia carcinomas within the United States. Am J Gastroenterol. 2004;99:582-588. doi:10.1111/j.1572-0241.2004. 04131.x.

20. Baquet CR, Commiskey P, Mack K, Meltzer S, Mishra SI. Esophageal cancer epidemiology in blacks and whites: racial and gender disparities in incidence, mortality, survival rates and histology. J Natl Med Assoc. 2005;97:1471-1478.

21. Wu X, Chen VW, Andrews PA, Ruiz B, Correa P. Incidence of esophageal and gastric cancers among Hispanics, non-Hispanic whites and non-Hispanic blacks in the United States: subsite and histology differences. Cancer Causes Control. 2007;18:585-593. doi:10.1007/s10552-007-9000-1.

22. Abrams JA, Fields S, Lightdale CJ, Neugut AI. Racial and ethnic disparities in the prevalence of Barrett's among patients who undergo upper endoscopy. Clin Gastroenterol Hepatol. 2008;6: 30-34. doi:10.1016/j.cgh.2007.10.006.

23. US census 2000. http://www.census.gov/census2000/states/nm. html. Accessed 1 April, 2008. 